
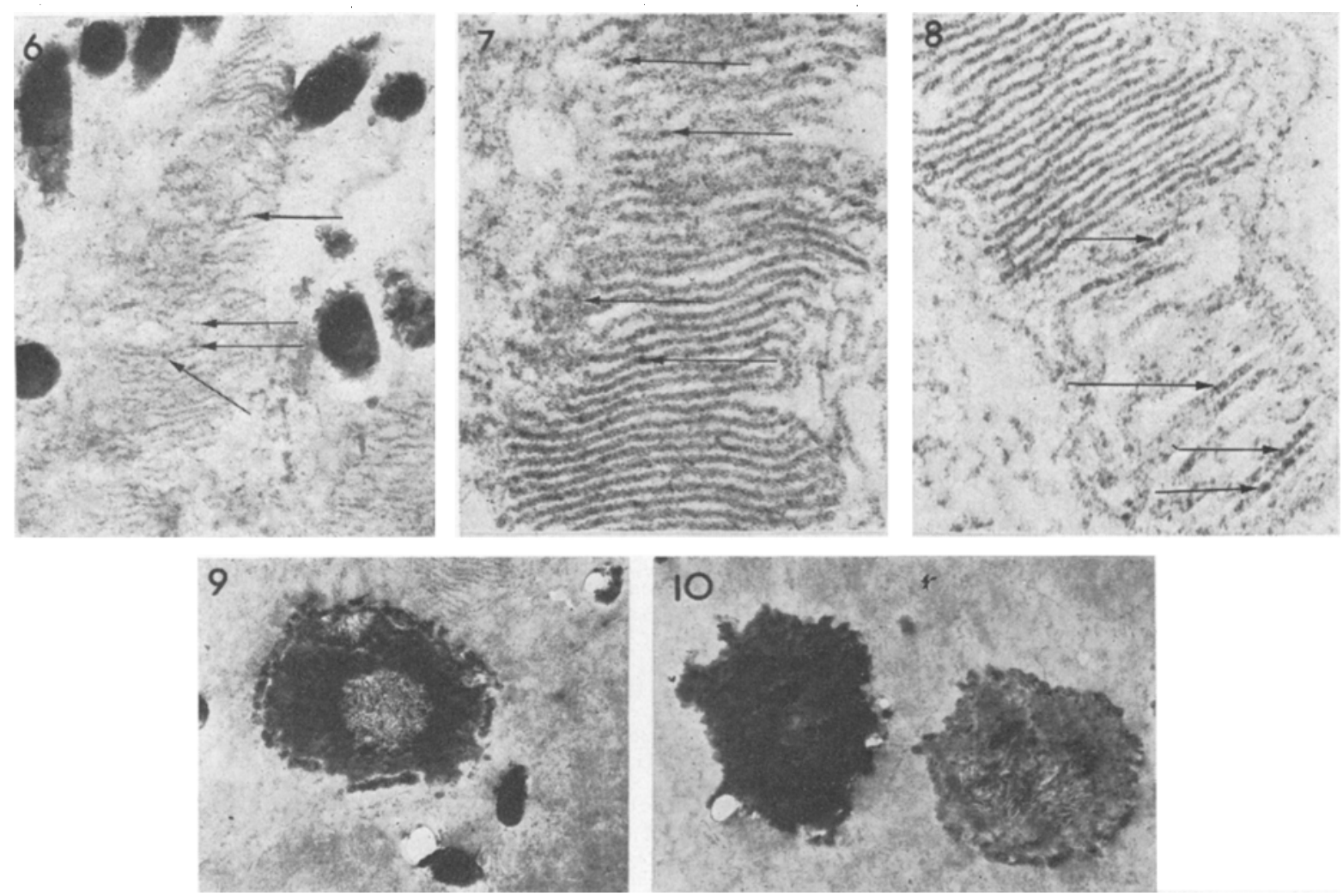

Figs. 6-10. 6. An outer segment of a stage 45 chick retina. Note a few very small and inconspicuous granular deposits (arrows) of ATPase positive sites. Unstained section, $\times 22,500.7$. and 8 . Outer segments of hatched chick retinas. Note increase in granular ATPase positive sites (arrows). Unstained sections, $\times 47,500.9$. and 10 . Retinal oil droplets at stage 45 . Note different intensity of ATPase localization. Unstained sections, $\times 13,500$.

(Figures 7 and 8). The inner segments only display ATPase positivity in certain oil droplets of both stages 45 and hatching. (Figures 9 and 10). According to the position of the droplets, they belong to those of the chief cones.

Discussion. Our results indicate an increase in ATPase positivity in Bruch's membrane and basal infolding layer at hatching, suggesting a possible increase in nutrient transport. Movement of nutrient across this region has been observed in the adults ${ }^{3,18,18}$.

Positive ATPase reactions in the outer segments of the adult retinas have been reported $\mathbf{1 , 7}$, and it was speculated that this enzyme may be related to rhodopsin. Our results indicate the initial presence of ATPase activity in the outer segments at stage 45 , which coincides in time with the first detected adult ERG ${ }^{10-12}$ and with the beginning of pupillary reflex ${ }^{20}$. This further implies that ATPase is important in the process of vision, possibly through its relationship with rhodopsin.

In the inner segments, positive ATPase sites are detected in chief cone oil droplets and not in other droplets, suggesting that different types of oil droplets may have different functions or metabolic activities.
The ATPase positive droplets probably serve to store ATP ${ }^{21}$.

Zusammenfassung. In der Retina des Hühnerembryos wurde im Zeitpunkt des Schlüpfens - sowohl in der Bruchschen Membran, wie auch im Pigmentepithel und der äusseren Stäbchenschicht - eine vermehrte ATPaseAktivität festgestellt.

\section{T. Yew ${ }^{22}$, H. M. Yoshimara and D. B. Meyer}

Department of Anatomy, Wayne State University, Detroit (Michigan 48207, USA), 20 January 1975.

18 W. LydA, N. ERICKSEN and N. KRISHNA, Am. J. ophthal. 44, 362 (1957).

19 Y. Nakaizumi, Arch. opthal. 72, 380 (1964).

${ }^{20}$ V. F. Lindeman, Am. J. Physiol. 148, 40 (1947).

${ }^{21}$ This work was supported by NIH grant No. EYOO477 from the National Eye Institute to DBM. The authors also wishes to thank Dr. G. KRAUs for his translation of the German Summary.

22 Present address: Dept. of Biology, New Asia College, Chinese University of Hong Kong, Shatin, N.T., Hong Kong.

\title{
A Comparison Between the Free Grafting of Sliced and Intact Muscles in the Rat ${ }^{1}$
}

The method of free grafting of entire mammalian muscles offers a number of advantages in the study of the physiology of muscle regeneration, but one drawback to this model is the persistence of a thin rim of original muscle fibres at the periphery of the graft ${ }^{2}$. This complicates the analysis of early contractile properties. On the other hand, the commonly used model of minced muscle regeneration ${ }^{3}$ has disadvantages due to the structural peculiarities, large amounts of connective tissue and low degree of recovery of muscle weight and tension development ${ }^{4}$.

In an effort to circumvent the disadvantages of both systems, a new experimental model was devised. It consists of slicing a muscle into a number of thin slabs, 
much like a sausage. In this way many of the architectural advantages of intact muscle grafts would remain whereas the repeated sectioning would ensure that no muscle fibres remained uninjured as contrasted to the peripheral rim of persisting original muscle fibres in intact (free) transplants. The results have shown that this is an efficient method for producing pure populations of regenerating muscle fibres.

Methods. 65 one-month-old male Wistar rats were used in this study. Either the extensor digitorum longus (EDL) or soleus (SOL) muscle was removed from one leg and cut transversely into 7 or 8 slices of approximately equal thickness. The muscle slices were then returned to their original muscle bed in as near to normal orientation as possible. At intervals from 4-60 days after implantation of the sliced muscles, the grafts were removed and analyzed in vitro by methods described previously ${ }^{2}$ for contraction time (time to peak) and tetanic tension. After determination of contractile properties, the muscles were frozen in liquid nitrogen, cross sectioned and stained for succinic dehydrogenase (SDH) and myosin adenosine triphosphatase (ATPase) at pH 9.4 as in our previous studies $^{5}$. In addition to selected specimens from the grafts studied above, a separate series of 16 sliced EDL grafts from 1-20 days after grafting was studied histologically.

Results. Histology. Despite attempts to replace the muscle slices in proper orientation, many of the slices had rotated within the muscle bed (presumably during the

Table I. Summary of histological events in regeneration of the sliced rat extensor digitorum longus muscle

\begin{tabular}{|c|c|}
\hline Days & Regenerative events \\
\hline $1 \sim 2$ & Myoblastic recruitment \\
\hline 3 & Early myotubes \\
\hline $4-5$ & Peak of myotube stage \\
\hline $6-7$ & $\begin{array}{l}\text { Peripheral myotubes develop into young muscles } \\
\text { fibres }\end{array}$ \\
\hline $7-14$ & $\begin{array}{l}\text { Myofibres align parallel to long axis of muscles. } \\
\text { Scattered poorly oriented myotubes still present in } \\
\text { central region }\end{array}$ \\
\hline $14+$ & Gradual histological maturation of muscle fibres \\
\hline
\end{tabular}

early hours after implantation) so that their fibre orientation was no longer parallel to the long axis of the original muscle. The histological results left no doubt that viable original muscle fibres had been eliminated from the sliced grafts and that contractile properties of the muscles, particularly during the first 2 weeks after slicing could be attributed to regenerating muscle fibres. All ischemic original muscle fibres in EDL slices have normally completely disappeared by the end of the 1st week. Table I summarizes the main regenerative events in sliced EDL grafts.

From the end of the 1st to early in the 3rd week postoperatively, the grafts contained small but consistent numbers of degenerating myotubes. These have also been seen in the regenerating minced EDL of the rat ${ }^{6}$ and are attributed to local denervation changes.

Contractile properties. The major difference between sliced and intact muscle grafts was the absence of contractile activity in sliced grafts 4 days postoperatively. Intact grafts of normal EDL or SOL muscles temporarily lose their ability to contract during the first 2 days after transplantation ${ }^{7}$ but by 4 days they contract weakly. The histology of 4-day intact grafts shows immature regenerating myotubes with a thin rim of old muscle fibres around the periphery ${ }^{8}$, and the contractile ability of 4day intact grafts has been attributed to the surviving (denervated) original muscle fibres. In sliced grafts, the lack of contractions at 4 days is correlated with an absence of surviving original muscle fibres and a population of myotubes too young to engage in significant contractile activity.

During later periods, the contractile properties of the sliced grafts are very similar to those of intact grafts (Table II). The contraction times of both SOL and EDL

1 Supported in part by a grant from the Muscular Dystrophy Associations of America, Inc.

${ }^{2}$ B. Carlson and E. Gutmann, Pflügers Arch., in press.

3 A. Stunitsky, Experimental Surgery of Muscles (Russian) (Izdatel. Akad. Nauk SSSR, Moskwa 1959).

B. Carlson and E. Gutmann, Expl. Neurol. 36, 239 (1972).

5 E. Gutmann and B. Carlson, Pflügers Arch, in press.

${ }^{6}$ B. CARLSON and E. GUTmann, unpublished data.

7 F. Vyskocil, B. Carlson and E. Gutmann, Pflügers Arch. 344, 181 (1973).

B. Carlson and E. Gutmann, Anat. Rec., in press.

Table II. Comparison of contraction time, tetanic tension and weight between sliced and intact grafts of the rat soleus and extensor digitorum longus muscles

\begin{tabular}{|c|c|c|c|c|c|c|c|}
\hline \multirow{3}{*}{ Day } & & \multicolumn{6}{|c|}{ Extensor digitorum longus } \\
\hline & & \multicolumn{2}{|c|}{ Contraction time (msec) } & \multicolumn{2}{|c|}{ Tetanic tension $(\mathrm{g})$} & \multicolumn{2}{|l|}{ Weight (mg) } \\
\hline & & Intact & Sliced & Intact & Sliced & Intact & Sliced \\
\hline & $(n=4)$ & $14.63 \pm 2.50$ & $-b$ & $1.21 \pm 0.03$ & none & - & - \\
\hline 7 & $(n=4)$ & $13.83 \pm 0.88$ & $16.38 \pm 2.01$ & $1.93 \pm 0.58$ & $1.53 \pm 0.51$ & - & $57.25 \pm 6.56$ \\
\hline 14 & $(n=10)$ & $17.63 \pm 1.17$ & $18.98 \pm 1.65$ & $5.45 \pm 0.53$ & $4.82 \pm 1.17$ & - & $36.70 \pm 5.36$ \\
\hline & $(n=9)$ & $14.28 \pm 0.20$ & $13.73 \pm 0.86$ & $13.50 \pm 3.57$ & $7.54 \pm 1.23$ & $59.40 \pm 7.26$ & $44.33 \pm 5.04$ \\
\hline & $(n=8)$ & $11.29 \pm 0.35$ & $12.97 \pm 0.53$ & $85.45 \pm 15.00$ & $15.61 \pm 3.35$ & $123.12 \pm 9.75$ & $84.13 \pm 15.31$ \\
\hline \multicolumn{8}{|c|}{ Soleus } \\
\hline & $(n=4)$ & $34.04 \pm 2.50$ & $-\mathrm{b}$ & $4.62 \pm 0.52$ & none & - & - \\
\hline & $(n=4)$ & $33.66 \pm 2.03$ & $35.82 \pm 3.54$ & $3.64 \pm 0.66$ & $0.35 \pm 0.23$ & - & $35.50 \pm 1.73$ \\
\hline & $(n=3)$ & $27.33 \pm 0.75$ & $33.93 \pm 2.02$ & $10.14 \pm 0.44$ & $2.86 \pm 1.91$ & - & $35.67 \pm 19.66$ \\
\hline & $(n=4)$ & $26.60 \pm 0.31$ & $22.15 \pm 12.79$ & $6.79- \pm \quad 0.78$ & $5.73 \pm 7.20$ & $52.00 \pm 5.63$ & $57.00 \pm 6.68$ \\
\hline & $(n==3)$ & $19.15 \pm 2.88$ & $22.77 \pm 1.42$ & $14.23 \pm 4.80$ & $8.83 \pm 5.68$ & $76.25 \pm 6.84$ & $56.67 \pm 10.41$ \\
\hline
\end{tabular}

a Data from B. CARtson and E. Gutmann, Pflügers Arch, in press. 'slight contraction (not measurable) after stimulus of 5 msec duration. 


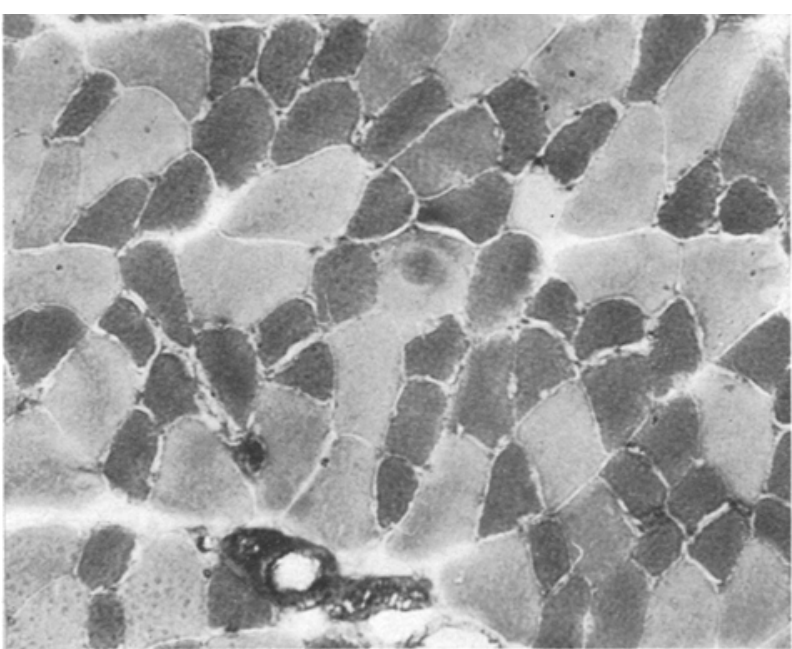

A

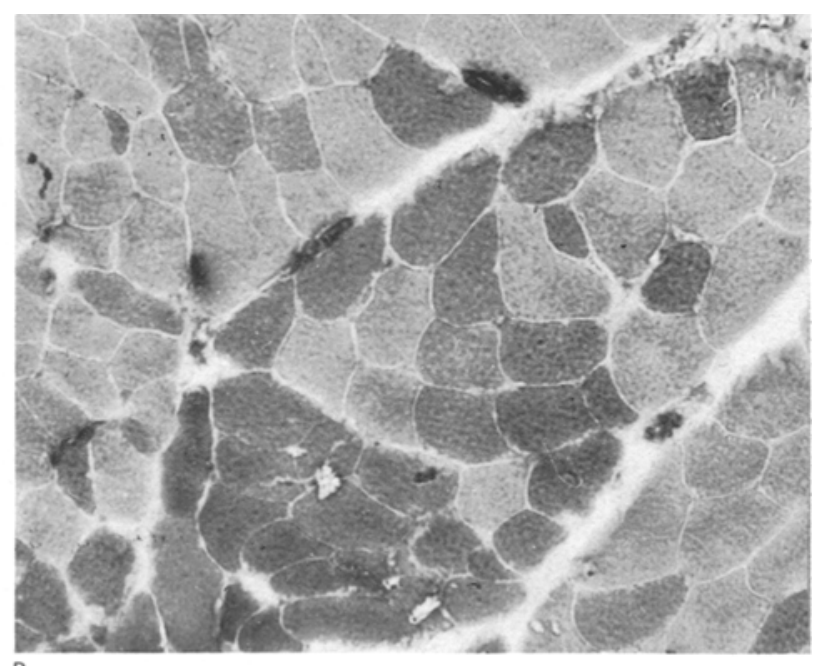

$B$

A) Normal rat extensor digitorum longus muscle stained for ATPase.

B) 60-day sliced extensor digitorum longus graft stained for ATPase. Distinct differences in fibre type staining are seen. Fibre type grouping rather than the normal checkerboard pattern is common.

grafts are relatively slow at first and speed up as the regenerating muscle fibres mature.

Tetanic tensions of sliced grafts were consistently less than those of intact grafts throughout the developmental period tested (Table II). These differences are a reflection of the gross appearance of the two types of grafts. Grafts of intact muscles are normally thicker and have a larger area of densely packed muscle fibres than do sliced grafts. Comparisons of weights are of little value because of variable amounts of connective tissue adherent to the muscles.

Histochemistry. The histochemical differentiation of muscle fibres within the sliced grafts followed a course typical of that seen in regenerating mammalian muscle. By 30 days differences in fibre types were beginning to emerge when sections were stained for either SDH or ATPase activity, and by 60 days the grafts were populated with distinct type I and type II fibres (Figure). As is the rule in regenerated and transplanted muscles, type grouping was commonly observed. Overall, the histochemical preparations revealed a close return of the regenerated muscle fibres to the normal condition.

Discussion. The method of transverse slicing has proven to be a useful model for studying regenerating mammalian muscle, and it represents another technique whereby an entire muscle can be regenerated or transplanted. Slicing is superior to mincing with respect to the greater amount of functional muscle that regenerates. Sliced muscle grafts are easier to analyze physiologically and structurally than grafts of intact muscles. Because original muscle fibres do not survive, the developmental gradients of contraction can be assumed to represent the properties of regenerating muscle fibres alone. The strength of contraction, however, is less than that of intact muscle grafts. This is probably due to the disruption of the internal architectural framework of the muscle by the slicing process. Thus as a physiological model slicing is a preferable technique of muscle grafting whereas intact muscle grafts produce muscles that are better from the clinical standpoint.

Summary. Transverse slicing is a new technique whereby a mammalian muscle can be freely grafted with success. This method eliminates contamination of the early graft by surviving muscle fibres and allows one to measure the development of contractile properties on a uniform population of regenerating muscle fibres.

\section{E. Gutmann and B. M. Carlson ${ }^{9}$}

Institute of Physiology, Czechoslovak Academy of Sciences, Budéjovická 1083,

Praha 4, KRC (Czechoslovakia), and

Department of Anatomy, 4622 MS II, University of Michigan, Ann Arbor (Michigan 48104, USA), 30 December 1974.

9 Part of this research was carried out while on a scientific exchange between the US and Czechoslovak Academies of Sciences.

\section{Glutamine Aminotransferase and Glutamine Aminohydrolase Ratio as a Possible Test for Antitumour Compounds}

Chakraborty et al. ${ }^{1}$, during their studies on the plasma ratio of glutamine aminotransferase (GAT) and glutamine aminohydrolase (GNase) in patients suffering from myeloproliferative diseases and different types of other blood disorders, had observed fluctuation in the ratio of these two enzymes with clinical and haematological conditions of the patients. They also found that the ratio was dependant upon the response of the patient to a particular drug therapy. Hence it was decided to see if the fluctuations in the plasma GAT/GNase ratio could be used as a test for the antitumour compounds. With this idea in view 3 of the known antitumour compounds were screened for their effect on the ratio of the two types

1 P. Chakraborty, M. Dutta Chawdhuri and G. C. Shrivastava, Indian. J. exp. Biol, in press (1974). 